\title{
Argon 39 als Reaktionsprodukt der Höhenstrahlung in Eisenmeteoriten
}

\author{
Von H. Wänke und Else Vilcsek \\ Aus dem Max-Planck-Institut für Chemie (Otto-Hahn-Institut), Mainz \\ (Z. Naturforschg. 14 a, 929—934 [1959]; eingegangen am 15. August 1959)
}

\begin{abstract}
Es wird die Abtrennung und Messung von Argon 39 beschrieben, das in Meteoriten durch Einwirkung der Höhenstrahlung auf die Eisenkerne entsteht. Von den drei untersuchten Eisenmeteoriten hatte nur der Meteorit Treysa meßbare Aktivitäten von Argon 39. Für den Zeitpunkt des Falles erhielten wir für Treysa eine Argon 39-Zerfallsrate von $0,36 \pm 0,02$ Zerfällen $/ \mathrm{kg}$ sec. Das negative Ergebnis für die beiden anderen untersuchten Meteorite Carbo und Clark County beweist, daß der Zeitpunkt des Falles dieser Meteorite mehr als 1000 Jahre zurückliegen muß.

Aus diesen Messungen konnte für Treysa ein Strahlungsalter von $6,0 \cdot 10^{8} \mathrm{Jahren}$ berechnet werden. Ferner ergibt sich für die Intensität der Höhenstrahlung für Teilchen mit Energien über $1 \mathrm{GeV}$ ein Wert von $I_{0}=0,25 \pm 0,07$ Teilchen $\mathrm{cm}^{-2} \mathrm{sec}^{-1}$ sterad $^{-1}$.
\end{abstract}

\section{a) Hochenergetische Kernreaktionen in Meteoriten}

Schon vor einigen Jahren wurde Helium als Reaktionsprodukt der Höhenstrahlung in Eisenmeteoriten nachgewiesen ${ }^{1}$. Sowohl Helium 3 als auch Helium 4 entstehen als Verdampfungsteilchen bei den Kernverdampfungsprozessen der Eisenkerne der Meteorite, die durch Teilchen der Höhenstrahlung ausgelöst werden. Außerdem erhält man als Folge dieser teilweisen Kernverdampfung Restkerne mit Massenzahlen unterhalb der des Eisens. Von diesen Kernen wurden zunächst wieder zwei Edelgase, nämlich Neon ${ }^{2}$ und Argon ${ }^{3}$ in Meteoriten aufgefunden. Kürzlich konnten als erste Vertreter fester Elemente auch Scandium ${ }^{4} 45$ sowie Kalium ${ }^{5} 40$ und 41 als Reaktionsprodukte der Höhenstrahlung in Meteoriten nachgewiesen werden. Ein Teil dieser Kerne entsteht möglicherweise nicht durch Kernverdampfung, sondern durch Fragmentation der Eisenkerne in zwei oder mehrere größere Bruchstücke.

Die Produktionsrate (Zahl der gebildeten Atome pro Gramm Meteorit und Sek.) eines beliebigen Kernes dieser Reaktionsprodukte kann man angeben, falls man den Erzeugungsquerschnitt dieses Kernes bei der Einwirkung von Protonen auf Eisen innerhalb des in Frage kommenden Energiebereichs, sowie die Intensität und die Energieverteilung der Höhenstrahlung kennt. Wir bezeichnen mit $\bar{\sigma}_{\mathrm{i}}$ den effektiven Erzeugungsquerschnitt des Kernes i für

1 F. A. Paneth, P. Reasbeck u. K. I. Mayne, Geochim. Cosmochim. Acta 2, 300 [1952].

2 P. Reasbeck u. K. I. Mayne, Nature, Lond. 176, 733 [1955].

3 W. Gentner u. J. Zähringer, Z. Naturforschg. 10 a, 498 [1955].

4 H. WäNKE, Z. Naturforschg. 13 a, 645 [1958].
Protonen mit einer Energieverteilung, wie sie der Primärkomponente der Höhenstrahlung entsprechen. Mit $W(E)$ für die Energieabhängigkeit des Wirkungsquerschnitts $\sigma_{\mathrm{i}}$; und mit $F(E) \mathrm{d}(E)$ für das differentielle Energiespektrum der Höhenstrahlung erhalten wir für den effektiven Wirkungsquerschnitt

$$
\bar{\sigma}_{\mathrm{i}}=\int_{E_{\min }}^{\infty} W(E) F(E) \mathrm{d} E / \int_{E_{\min }}^{\infty} F(E) \mathrm{d} E
$$

und für die Produktionsrate $H_{\mathrm{i}}$

$$
H_{\mathrm{i}}=4 \pi \bar{\sigma}_{\mathrm{i}} I_{0} L / A,
$$

wobei $L$ die Loschmidssche Zahl, $A$ das Atomgewicht des Eisens und $I_{0}$ die Intensität der Höhenstrahlung in Teilchen $\mathrm{cm}^{-2} \mathrm{sec}^{-1}$ sterad ${ }^{-1}$ ist. Die Zahl der Atome eines Reaktionsproduktes pro Gramm Meteorit ist dann gegeben durch

$$
N_{\mathrm{i}}=H_{\mathrm{i}} T=4 \pi \bar{\sigma}_{\mathrm{i}} \cdot I_{0} T L / A .
$$

Hierin bedeutet $T$ das Strahlungsalter der Meteoriten, das ist die Zeit, während der die Atomkerne des Meteoriten der Höhenstrahlung ausgesetzt sind, also die Zeit seit dem Ausbrechen des Meteoriten aus einem größeren Himmelskörper. Mißt man nun die Konzentration irgendeines Reaktionsproduktes, so kann man aus obiger Gleichung das Strahlungsalter $T$ ausrechnen. Dies ist auch schon öfters versucht worden ${ }^{6-9}$, doch blieben alle diese Berechnungen sehr unsicher, da über die Intensität der Höhenstrahlung im freien Weltraum, insbesondere bezüglich von

\footnotetext{
5 H. Voshage u. H. Hintenberger, Z. Naturforschg. 14 a, 194 [1959].

6 G. R. Martin, Geochim. Cosmochim. Acta 3, 288 [1953].

${ }^{7}$ E. L. Fireman, Phys. Rev. 97, 1303 [1955].

8 L. A. Currie, W. F. Libby u. R. L. Wolfgang, Phys. Rev. 101, 1557 [1956].

9 K. H. Ebert u. H.Ẅ̈nKe, Z. Naturforschg. 12 a, 766 [1957].
} 
Teilchen mit Energien unter $1 \mathrm{GeV}$, nur wenig bekannt ist. Voraussetzung für alle diese Überlegungen ist natürlich eine räumliche und zeitliche Konstanz der Höhenstrahlung.

Alle unsere bisherigen Betrachtungen gelten nur für kleine Meteorite, für die man die Absorption der Höhenstrahlungsteilchen im Meteoriten sowie den Einfluß von Sekundärteilchen vernachlässigen kann. Für größere Meteorite hat man an Stelle von $I_{0}$ den Fluß der primären und sekundären Teilchen zu setzen, ebenso muß dann für die Berechnung des effektiven Wirkungsquerschnittes die Energieverteilung aller in Frage kommenden Teilchen berücksichtigt werden.

Einen großen Schritt vorwärts gelangt man, wenn man neben den Messungen an stabilen Kernen auch solche an radioaktiven Reaktionsprodukten ausführt. Im Zeitpunkt des Falles des Meteoriten ist die Zahl $A_{\mathrm{k}}$ der pro Zeiteinheit und Gramm Meteorit zerfallenden Atomkerne $\mathrm{k}$ gleich der Zahl der pro Zeiteinheit und Gramm gebildeten Kerne, da man fast immer Sättigung voraussetzen kann:

$$
A_{\mathrm{k}}=4 \pi \bar{\sigma}_{\mathrm{k}} I_{0} L / A \text {. }
$$

Dividiert man Gl. (3) durch Gl. (4), so erhält man nach $T$ aufgelöst, für das Strahlungsalter

$$
T=\frac{\bar{\sigma}_{\mathrm{k}}}{\sigma_{\mathrm{i}}} \frac{N_{\mathrm{i}}}{A_{\mathrm{k}}},
$$

Die Kenntnis des Energiespektrums der erzeugenden Teilchen ist jedoch nicht notwendig, sofern es sich um Reaktionsprodukte handelt, deren Erzeugungsquerschnitte innerhalb des in Frage kommenden Energiebereichs annähernd konstant sind. Für die Berechnung des Strahlungsalters wird der Bruch $\sigma_{\mathrm{k}} / \sigma_{\mathrm{i}}$ identisch mit $\sigma_{\mathrm{k}} / \sigma_{\mathrm{i}}$ für eine beliebige Energie, falls die Erzeugungsquerschnitte der beiden betrachteten Kerne i und k die gleiche Energieabhängigkeit besitzen. In diesen beiden letzten Fällen gilt dann Gl. (5) allgemein, also auch für "große“ Meteorite, da ja dann keine Größen auftreten, die von der Energieverteilung der Teilchen, die die Reaktionen auslösen, abhängig sind, und die Intensität dieser Teilchen in Gl. (5) nicht eingeht. Die ersten Messungen eines durch die Höhenstrahlung induzierten

10 E. L. Fireman u. D. Schwarzer, Geochim. Cosmochim. Acta 11, 252 [1957].

11 F. Begemann, J. Geiss u. D. C. Hess, Phys. Rev. 107, 540 [1957].

12 F. Begemann, P. Eberhardt u. D. C. Hess, Z. Naturforschg. 14 a, 500 [1959]. radioaktiven Kernes in Meteoriten stammen von Fireman und Schwarzer ${ }^{10}$. Diese Autoren konnten 1957 Tritium in Eisenmeteoriten nachweisen. Weitere Tritiummessungen wurden von BEGEMANN et al. ${ }^{11,12}$ und von Goebel und Schmidlin ${ }^{13}$ für eine Reihe von Steinmeteoriten ausgeführt. Aus diesen Messungen konnten für die vier insgesamt untersuchten Steinmeteorite $\mathrm{T} \cdot{ }^{3} \mathrm{He}$-Strahlungsalter von $13 \cdot 10^{6}$ bis $280 \cdot 10^{6}$ Jahren berechnet werden. Von allen in Frage kommenden aktiven Kernen besitzt Tritium den weitaus größten Erzeugungsquerschnitt; die Halbwertszeit von 12,3 Jahren beschränkt diese Methode jedoch auf in jüngerer Zeit gefallene Meteorite.

Im Jahre 1957 berichteten Kohman und Ehmann ${ }^{14}$ über die Auffindung von Beryllium $10\left(T_{1 / 2}=2,7 \cdot 10^{6}\right.$ Jahre $)$, Aluminium $26\left(T_{1 / 2}=0,8 \cdot 10^{6}\right.$ Jahre $)$ und Kobalt $60\left(T_{1 / 2}=5,2\right.$ Jahre $)$ in Meteoriten. In allerletzter Zeit konnte von Fireman ${ }^{15}$ erstmals Argon 39 in dem 1947 gefallenen Meteoriten Sichote Alin aufgefunden werden. Argon 39 hat eine Halbwertszeit von 260 Jahren, so daß Messungen an einer größeren Zahl von Meteoriten möglich sind. Die zu erwartenden Aktivitäten sind allerdings um etwa eine Größenordnung kleiner als die von Tritium. Für Messungen von Argon 39 erschien uns der Meteorit Treysa besonders geeignet, da seit dem Zeitpunkt seines Falles (1916) nur wenig Argon 39 zerfallen ist und außerdem wegen des hohen Gehaltes dieses Meteoriten an Helium und Neon auch größere Aktivitäten von Argon 39 zu erwarten waren. Die Meteorite Carbo und Clark County sind Funde; über den Zeitpunkt ihres Falles ist nichts bekannt. An allen drei Meteoriten sind aus den gleichen Proben auch Bestimmungen von Beryllium 10, Aluminium 26 und Chlor 36 vorgesehen, doch ist die Abtrennung und Reinigung dieser Isotope noch im Gange.

\section{b) Extraktion von Argon 39}

Eine schematische Darstellung der verwendeten Hochvakuumapparatur gibt Abb. 1. Die Proben wurden in Form von Frässpänen in den seitlichen Ansatz des Lösungskolbens $\mathrm{L}$ eingebracht. Im Kolben befand sich eine schwefelsäurehaltige gesättigte Quecksilbersulfatlösung, die als Lösungsflüssigkeit für die Meteoritproben diente.

13 K. Goebel u. P. Schmidlin, Geochim. Cosmochim. Acta (1959), im Druck.

14 T. P. Kohman u. W. D. Ehmann, UNESCO/NS/RIC/219.

15 E. L. Fireman, Nature, Lond. 181, 1613 [1958]. 


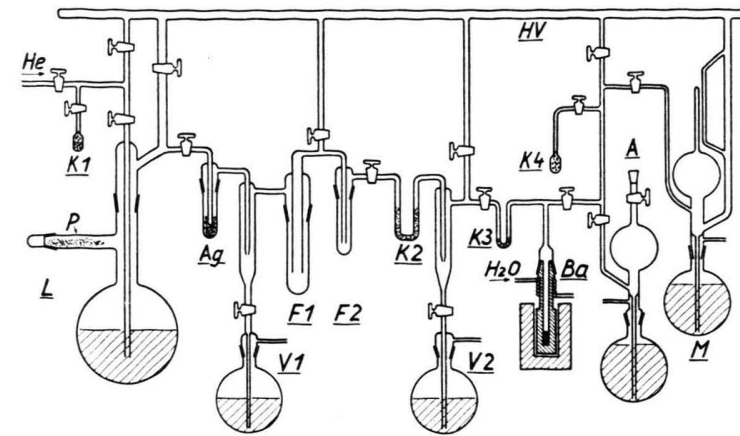

Abb. 1. Hochvakuumapparatur zur Extraktion und Reinigung von Argon 39 aus Eisenmeteoriten.

Der Lösungskolben wurde unter mehrmaligem Spülen mit reinstem Helium gut evakuiert. Vor dem Lösen der Proben wurde über die Aktivkohle $\mathrm{K}_{1}$ eine gemessene Menge Argon (ca. 0,4 $\mathrm{cm}^{3}$ NPT) in den Gasraum des Kolbens eingeleitet, worauf die Meteoritspäne mit Hilfe eines Magneten in kleinen Portionen aus dem seitlichen Ansatz in den Kolben befördert wurden. Während des Lösens wurde der Kolben mit Wasser gekühlt; das Lösen von etwa $100 \mathrm{~g}$ Meteorit erforderte ca. $1^{1 / 2} \mathrm{~h}$. Die von uns verwendete Lösungsflüssigkeit hatte den Vorteil, nur kleine Mengen Wasserstoff in Freiheit zu setzen, so daß es nur zu einem geringen Druckanstieg im Kolben kam. Nach vollständiger Lösung der Proben wurde gut mit Helium gespült und dieses Helium zusammen mit den freigesetzten Gasen und dem Träger-Argon durch eine Reihe von Trennstufen hindurchgeleitet. Zunächst passierten die Gase eine kleine Falle mit Silberwolle, die etwa freigesetzten Chlorwasserstoff binden sollte. (Diese Vorkehrung war notwendig, da auch Chlor abgetrennt werden sollte, zur Messung des gleichfalls in Meteoriten vorhandenen Chlor 36.) Das dahintergeschaltete Quecksilberventil war gegen den Lösungskolben zu für einen Überdruck von ca. 5-10 mm eingestellt, um nicht zu große Wasserdampfmengen in die nun folgenden mit flüssigem Stickstoff gekühlten Kühlfallen $F_{1}$ und $F_{2}$ übertreten zu lassen. Im weiteren Verlauf wurde das Gasgemisch durch ein mit Aktivkohle gefülltes U-Rohr geleitet, das ebenfalls in flüssigen Stickstoff tauchte. Daran angeschlossen war ein zweites Quecksilberventil, für eine Druckminderung von etwa $2 \mathrm{~mm}$. Die dieses zweite Ventil passierenden Gase wurden ständig abgepumpt. Nachdem der Lösungskolben mehrmals mit insgesamt etwa $400 \mathrm{~cm}^{3}$ Helium durchgespült worden war, wurde das Quecksilberventil 2 vollständig geöffnet, und so die Aktivkohle $\mathrm{K}_{2}$ für $5 \mathrm{~min}$ mit dem Hochvakuum verbunden. Dabei desorbierte Helium und ein großer Teil des Wasserstoffs, das Argon blieb jedoch an der Kohle. Der zur Hochvakuumleitung führende Hahn wurde daraufhin geschlossen, die Aktivkohle $\mathrm{K}_{2}$ auf $60{ }^{\circ} \mathrm{C}$ aufgeheizt und die hier bisher adsorbierten Gase an die Kohle $K_{3}$, die mit flüssigem Stickstoff gekühlt war, gebracht, die Aktivkohle $\mathrm{K}_{3}$ mit dem McLeodManometer verbunden und die dabei desorbierten letzten Helium- und Wasserstoffreste abgepumpt. Dieser Vorgang wurde noch zweimal wiederholt. Die an der Aktiv- kohle $\mathrm{K}_{3}$ adsorbierten $\mathrm{Gase}$ wurden bei $+60{ }^{\circ} \mathrm{C}$ desorbiert, der Bariumofen $\mathrm{Ba}$ für $30 \mathrm{~min}$ auf $800{ }^{\circ} \mathrm{C}$ aufgeheizt und anschließend auf etwa $300^{\circ}$ abgekühlt. Dabei wurden alle unedlen Gase durch das Barium gebunden und somit aus dem Gemisch entfernt. Das zurückbleibende Argon wurde in das McLeod-Manometer gebracht und gemessen. Die Ausbeute war in allen Fällen höher als 97\%, während in Leerversuchen ohne Träger-Argon stets nur vernachlässigbar kleine Mengen Argon nachzuweisen waren. Das Argon wurde nun durch Adsorption und Desorption an der Aktivkohle $\mathrm{K}_{4}$ in die Abfülvorrichtung $\mathrm{A}$, die als Töpler-Pumpe ausgebildet war, übergeleitet und in die Meßküvette gebracht.

\section{c) Bestimmung der Aktivitäten}

Die Aktivitätsmessungen erfolgten mittels eines Glokkenzählrohres (Fenster Mylar $0,9 \mathrm{mg} / \mathrm{cm}^{2}$ ), das zur Verringerung des Nulleffektes in Antikoinzidenz mit einem umgebenden Kranz von Schirmzählrohren geschaltet war. Die Meßküvetten (Abb. 2) bestanden aus Plexiglas, de-

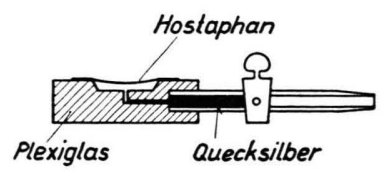

Abb. 2. Meßküvette aus Plexiglas zur Bestimmung der Aktivitäten.

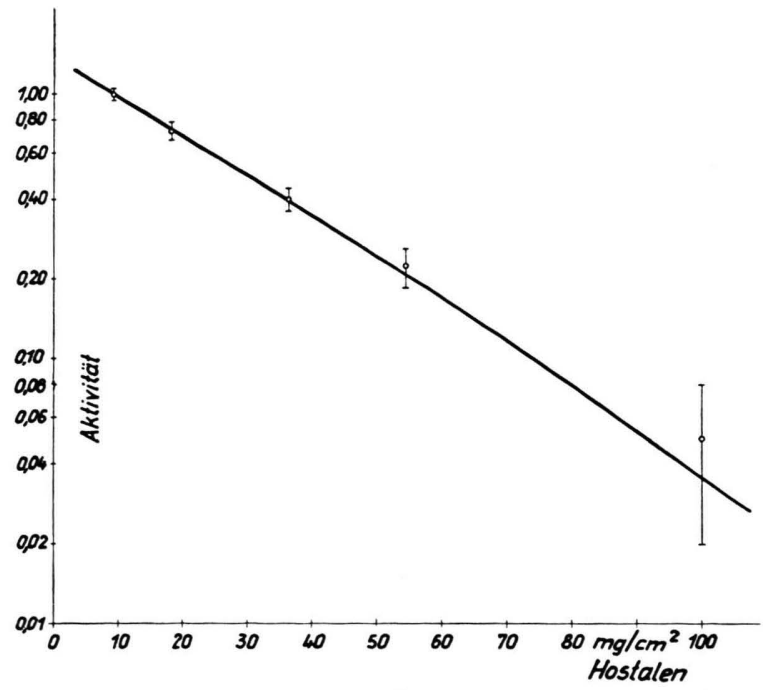

Abb. 3. Absorptionsmessungen des aus dem Meteoriten Treysa extrahierten Argon 39 ( $\bigcirc$ Meßpunkte mit Fehlergrenzen von Argon 39 aus Treysa. Ausgezogene Kurve: aus Messungen einer großen Aktivität von künstlich erzeugtem Argon 39 erhalten).

ren zylindrischer Hohlraum zur Aufnahme des zu messenden Gases einen mittleren Durchmesser von $22 \mathrm{~mm}$ und ein Volumen von ca. 1,2 $\mathrm{cm}^{3}$ besaß. Gegen das Zählrohr waren die Küvetten mit einer Polyester-Folie (Hostaphan) von $7 \mathrm{mg} / \mathrm{cm}^{2}$ abgeschlossen. Die von uns verwendete Methode der Aktivitätsmessung hatte den großen 
Vorteil, daß mit Hilfe von Absorbern auch Vergleiche der Strahlungsenergie durchgeführt werden konnten.

Abb. 3 zeigt die mit verschiedenen Absorbern (Polyäthylen $=$ Hostalen) gemessenen Werte der aus dem Meteoriten Treysa abgetrennten Aktivität im Vergleich mit einer größeren, unter gleichen Bedingungen gemessenen Argon 39-Aktivität, die durch Bestrahlung von Kalium im Reaktor durch die Reaktion ${ }^{39} \mathrm{~K}(\mathrm{n}, \mathrm{p}){ }^{39} \mathrm{~A}$ erhalten worden war. Die ausgezogene Kurve wurde durch Messungen des Vergleichsargon erhalten; der besseren Übersicht wegen sind hierfür keine Meßpunkte bzw. Fehlergrenzen eingetragen. Da es sich hierbei aber um Zählraten von etwa $1000 \mathrm{Imp} / \mathrm{min}$ handelte, sind diese Fehler vernachlässigbar klein. Die vollkommene Übereinstimmung der Absorptionskurven ist ein sicherer Beweis dafür, daß es sich bei den aus den Meteoritproben extrahierten Aktivitäten tatsächlich um Argon 39 handelt. Die absoluten Aktivitätseichungen wurden mit Natrium 22 und Strontium 90 durchgeführt, da diese beiden Isotope etwa dieselbe Maximalenergie wie Argon 39 besitzen und überdies ein recht ähnliches Energiespektrum zeigen. Natrium 22 und Strontium 90 entstammten Standardlösungen, deren absolute Aktivitäten durch Messungen in einem $4 \pi$-Zähler bestimmt wurden. Die Fehler dieser Eichungen betragen kaum mehr als 5\%. Für die Zählausbeute erhielten wir auf diese Weise einen Wert von $0,248 \pm 0,012$.

\section{d) Diskussion der Ergebnisse}

Wie aus Tab. 1 ersichtlich, konnten nur in dem 1916 gefallenen Meteoriten Treysa meßbare Aktivi- täten von Argon 39 aufgefunden werden. Aus den Bestimmungen 6 bis 9 ergibt sich für Treysa eine mittlere Zählrate von $0,48 \pm 0,02 \mathrm{Imp} / \mathrm{min}$ für $100 \mathrm{~g}$ Meteorit. Unter Berücksichtigung der Zählausbeute erhalten wir somit eine Zerfallsrate von $0,32 \pm 0,02$ Zerfälle ${ }^{39} \mathrm{~A} / \mathrm{kg} \mathrm{sec}^{*}$. Daraus errechnet sich zum Zeitpunkt des Falles

Aktivität Argon $39=0,36 \pm 0,02$ Zerfälle $/ \mathrm{kg}$ sec. Die maximalen Argon 39-Zählraten für Carbo und Clark County betragen nur etwa $4 \%$ bzw. $2 \%$ der für Treysa gefundenen Werte. Da diese Meteorite gegenüber Treysa etwa vergleichbare Mengen Helium, Neon und Scandium enthalten, folgt daraus, daß der Zeitpunkt des Falles für Carbo und Clark County mindestens 4 bis 5 Halbwertszeiten $\left(T_{1 / 2}\right.$ $=260$ Jahre) von Argon 39, d. h. mehr als 1000 Jahre zurückliegen muß.

Wie aus Arbeiten von Fireman und Zähringer ${ }^{16}$ sowie Schaeffer und Zähringer ${ }^{17}$ zu entnehmen ist, ist der Erzeugungsquerschnitt für Argon 37 bei der Einwirkung von Protonen auf Eisen in einem Energiebereich von $430 \mathrm{MeV}$ bis $6 \mathrm{GeV}$ nahezu konstant. Ähnliche Verhältnisse darf man sicherlich auch für Argon 39 annehmen, da die Erzeugungsquerschnitte für Kerne mit Massenzahlen, die etwa 10-15 Masseneinheiten unter der Massenzahl des getroffenen

\begin{tabular}{|c|c|c|c|c|c|c|c|}
\hline & Probe & $\begin{array}{c}\text { Einwaage } \\
\text { g }\end{array}$ & $\begin{array}{c}\text { Argon- } \\
\text { ausbeute } \\
\%\end{array}$ & $\begin{array}{l}\text { Zählrate }+\mathrm{NE} \\
\operatorname{Imp} / \min \end{array}$ & $\begin{array}{l}\text { Nulleffekt } \\
\text { Imp/min }\end{array}$ & $\begin{array}{l}\text { Nettozählrate } \\
\text { Imp/min }\end{array}$ & $\begin{array}{c}\text { Zählrate f. } 100 \mathrm{~g} \\
\text { Einwaage und } \\
100 \% \text { Ausbeute } \\
\text { Imp/min }\end{array}$ \\
\hline 1 & Normales Eisen & 100 & 102 & $0,63 \pm 0,02$ & $\begin{array}{l}0,63 \pm 0,02 \\
0,64+0,02\end{array}$ & $0,00 \pm 0,03$ & $\leq 0,03$ \\
\hline 2 & Carbo & 170 & 100 & $0,66 \pm 0,02$ & $0,68 \pm 0,02$ & $0,00 \pm 0,03$ & $\leq 0,02$ \\
\hline 3 & Clark County & 90 & 99 & $0,63 \pm 0,02$ & $\begin{array}{l}0,06 \pm 0,02 \\
0,64 \pm 0,02 \\
0,63 \pm 0,03\end{array}$ & $-0,02 \pm 0,03$ & $\leq 0,01$ \\
\hline 4 & & 98 & 98 & & $0,64+0,03$ & $0,42 \pm 0,05$ & $0,44 \pm 0,05$ \\
\hline 5 & Treysa 2 & 99 & 99 & $1,12 \pm 0,04$ & $0,65+0,02$ & $0,47 \pm 0,05$ & $0,48 \pm 0,05$ \\
\hline 6 & Treysa $1+2$ & 197 & 96 & $1,57 \pm 0,05$ & $0,64 \pm 0,03$ & $0,92 \pm 0,06$ & $0,49 \pm 0,03$ \\
\hline 7 & , & 197 & 93 & $1,53 \pm 0,04$ & $0,66 \pm 0,03$ & $0,88 \pm 0,05$ & $0,48 \pm 0,03$ \\
\hline 8 & , & 197 & 92 & $1,49 \pm 0,05$ & $\begin{array}{l}0,66 \pm 0,03 \\
0,68 \pm 0,03\end{array}$ & $0,83 \pm 0,06$ & $0,46 \pm 0,03$ \\
\hline 9 & , & 197 & 90 & $1,54 \pm 0,03$ & $0,63 \pm 0,03$ & $0,88 \pm 0,04$ & $0,50 \pm 0,02$ \\
\hline
\end{tabular}

Tab. 1. Zählraten der aus den Meteoritproben extrahierten Aktivitäten.

* Einer privaten Mitteilung von Fireman zufolge fanden E. L. Fireman und J. De Felice beim Meteoriten Treysa für Argon 39 eine Zerfallsrate von 0,222 Zerfällen $/ \mathrm{kg}$ sec.
16 E. L. Fireman u. J. Zähringer, Phys. Rev. 107, 1695 [1957]. 17 O. A. Schaeffer u. J. Z̈̈HRINGer, Z. Naturforschg. 13 a, 346 [1958]. 
Kernes liegen, nur geringe Energieabhängigkeit zeigen ${ }^{18-21}$. Für Argon 39 erhält man aus diesen Arbeiten innerhalb des erwähnten Energiebereichs einen mittleren Erzeugungsquerschnitt von $5,1 \mathrm{mb}$. Am zweckmäßigsten für die Berechnung des Strahlungsalters aus der Argon 39-Aktivität wäre natürlich ein Vergleich mit der Konzentration des stabilen Argon 38. Leider ist der Gehalt des Meteoriten Treysa an Argon 38 bisher nicht bekannt, doch sind solche Messungen für die nahe Zukunft von uns geplant. Der beste derzeit mögliche Vergleich ist der mit Scandium 45 4, 22. Der Erzeugungsquerschnitt von Scandium 45 bei der Bestrahlung von Eisen beträgt für Protonen mit Energien zwischen $340 \mathrm{MeV}$ und $6 \mathrm{GeV}^{18-21}$ ca. $21 \mathrm{mb}$. Die Zahl der durch die Höhenstrahlung erzeugten Atome Scandium 45 pro Gramm Meteorit fand sich für Treysa im Mittel zu $N_{45 \mathrm{Sc}}=2,8 \cdot 10^{13}$ Atome/g. Wir können nun in Gl. (5) einsetzen und erhalten für das Strahlungsalter des Meteoriten Treysa den Wert von 6,0 $10^{8}$ Jahren. Dieses Strahlungsalter liegt somit wesentlich höher als die Werte der Strahlungsalter von Steinmeteoriten ${ }^{10-13}$. Die Erzeugungsquerschnitte der Kerne Argon 39 und Scandium 45 sind nur mit einer Genauigkeit von etwa $20 \%$ bekannt, dem von uns berechneten Strahlungsalter haftet demnach ebenso ein Fehler von etwa $20 \%$ an.

Der Meteorit Treysa besitzt ein Gesamtgewicht von $63 \mathrm{~kg}$. Die Proben erhielten wir durch Abfräsen einer ebenen Fläche, die etwa einen halben Radius vom Mittelpunkt des annähernd kugelförmigen Meteoriten entfernt lag. Aussagen über die Intensität der Höhenstrahlung sind im Falle dieses Meteoriten ohne Berücksichtigung der Schwächung der Primärteilchen und des Einflusses von Sekundärteilchen nicht möglich. Wie an anderer Stelle näher ausgeführt wird ${ }^{9,22}$, läßt sich jedoch mit Hilfe der Neon-Isotope, die ebenfalls Reaktionsprodukte der Höhenstrahlung sind, der Anteil der Sekundärteilchen an den hochenergetischen Reaktionen mit den Eisenkernen bestimmen, da Neon nur mit Energien von etwa

18 R. E. Batzed, D. R. Miller u. G. T. Seaborg, Phys. Rev. 84, 671 [1951].

19 A. P. Vinogradov, I. P. Alimarin, V. I. Baranov, A. K. Lavrukhina, T. V. Baranova u. F. I. Pavlotskaya, Session of the Academy of Sciences of the USSR on the Peaceful Uses of Atomic Energy, July 1955, S. 85 (Englische Übersetzung).

20 G. Friedlander, J. M. Miller, R. Wolfgang, J. Hudis u. E. BaKer, Phys. Rev. 94, 727 [1954].

21 D. W. Barr, Thesis, University of California 1957.
$1 \mathrm{GeV}$ aufwärts mit merklichen Erzeugungsquerschnitten gebildet wird. Aus verschiedenen Bestrahlungsexperimenten und den von Caretto, Hudis und Friedlander ${ }^{23}$ gemessenen Werten für die Produktion von Natrium 24 bei der Bestrahlung von Kupfer mit Protonen zwischen 1 und $6 \mathrm{GeV}$ sowie dem von Winckler, Stix, Dwight und $\mathrm{S}_{\mathrm{ABIN}}{ }^{24}$ angegebenen Ansatz für das differentielle Energiespektrum der primären Höhenstrahlungsteilchen errechnet sich für Neon 22 ein totaler effektiver Erzeugungsquerschnitt von $\sigma_{22 \mathrm{Ve}}=5,0 \mathrm{mb}$. Für das Verhältnis der Produktionsraten von Scandium 45 zu Neon 22 ergibt sich somit $21 / 5,0=4,2$. Das Verhältnis der tatsächlich gefundenen Konzentrationen beträgt für Treysa $16,5^{22,25}$. (Der absolute Neongehalt wurde mit dem gleichen, zu den Argon 39-Messungen verwendeten Probenmaterial nochmals bestimmt. Hierbei ergab sich $18,5 \cdot 10^{-8} \mathrm{~cm}^{3} \mathrm{Ne} \mathrm{NPT} / \mathrm{g}$.) Der Überschuß von Scandium 45 kann also nur durch Teilchen mit Energien unter $1 \mathrm{GeV}$ verursacht worden sein. Tatsächlich liegen die Energien der Sekundärteilchen im Mittel unter $700 \mathrm{MeV}$. Der Anteil der Primärteilchen mit Energien über $1 \mathrm{GeV}$ an der Produktion von Scandium 45 beträgt somit $25 \%$, die restlichen $75 \%$ werden durch Sekundärteilchen erzeugt, wenngleich eine Erzeugung durch im freien Weltraum möglicherweise vorhandene Primärteilchen mit Energien unter $1 \mathrm{GeV}$ nicht ausgeschlossen werden kann. Denselben Anteil der Primärteilchen mit Energien über $1 \mathrm{GeV}$ kann man nach dem oben Gesagten auch für die Erzeugung von Argon 39 annehmen. Unter Berücksichtigung des Massenverlustes des Meteoriten beim Durchgang durch die Atmosphäre $\left(R=0,6 R_{0}\right){ }^{9}$ ergibt sich für unsere Proben ein mittlerer Schwächungsfaktor der Höhenstrahlungsteilchen durch Absorption von 0,48. Setzt man nun in Gl. (4) ein, so erhält man für die Intensität der Höhenstrahlung für Teilchen mit Energien über $1 \mathrm{GeV}$ einen Wert von $I_{0}=0,25$ Teilchen $\mathrm{cm}^{-2} \mathrm{sec}^{-1}$ sterad $^{-1}$. Dies ist aber genau derselbe Wert, wie er in Experimenten mit Kernphotoplatten am Gipfel der Atmosphäre gefunden wurde ${ }^{26}$. Die

${ }^{22} \mathrm{H}$. WäNKE, In Vorbereitung, erscheint voraussichtlich in $\mathrm{Z}$ Naturforschg.

23 A. A. Caretto, J. Hudis u. G. Friedlander, Phys. Rev. 110, 1130 [1958].

24 J. R. Winckler, T. Stix, K. Dwight u. R. Sabin, Phys. Rev. 79, 656 [1950].

25 H. Wänke u. H. Hintenberger, Z. Naturforschg. 13 a, 895 [1958].

${ }_{26}$ L. H. Meredith, J. A. van Allen u. M. B. Gotrlieb, Phys. Rev. 99, 198 [1955]. 
absolute Übereinstimmung ist allerdings reiner $\mathrm{Zu}$ fall, denn wenngleich die einzelnen Reaktionsprodukte der Höhenstrahlung in den Meteoriten nun auf etwa $5 \%$ genau bekannt sind, so stecken in den Werten für die Erzeugungsquerschnitte doch Fehler bis etwa $20 \%$. Etwa in dieser Größe liegt auch die Unsicherheit bezüglich der Massenverluste der Meteorite beim Durchgang durch die Erdatmosphäre. Doch ist auf jeden Fall hiermit ein weiterer Beweis dafür geliefert, daß es möglich ist, mit Hilfe der Meteorite recht zuverlässige Angaben bezüglich der Intensität der Höhenstrahlung zu gewinnen. Von besonderem Interesse wären Messungen an sehr kleinen Meteoriten (unter etwa $1 \mathrm{~kg}$ ), da für diese die Sekundär- prozesse vernachlässigbar werden und somit sofort Aussagen über das Vorhandensein von Primärteilchen mit Energien unter $1 \mathrm{GeV}$ möglich würden.

Besonderen Dank möchten wir auch an dieser Stelle für die Überlassung der Meteoritproben aussprechen. Die Proben vom Meteoriten Treysa erhielten wir von Professor H. Winkler (Marburg); Carbo entstammte der Sammlung Professor Paneths und Clark County erwarben wir von Dr. H. H. Nininger.

Für ihre Mithilfe bei der Durchführung der Experimente danken wir Fräulein U. SchEerer.

Die Arbeit wurde unterstützt durch Sachbeihilfen des Bundesministeriums für Atomkernenergie und Wasserwirtschaft.

\title{
Das Temperaturverhalten des Isotopieeffektes bei Stromdurchgang in flüssigen Alkalimetallen
}

\author{
Von A. Lodding \\ Aus dem Institut für Physik, Chalmers Technische Hochschule, Göteborg \\ (Z. Naturforschg. 14 a, 934-937 [1959] ; eingegangen am 2. Mai 1959)
}

\begin{abstract}
Die Temperaturabhängigkeit des sog. HAEFFner-Effektes wurde in Kalium zwischen $79{ }^{\circ} \mathrm{C}$ und $335^{\circ} \mathrm{C}$ untersucht. Es ergab sich ein Anstieg des Masseneffektes $\mu$ bis ca. $250{ }^{\circ} \mathrm{C}$; oberhalb $300^{\circ} \mathrm{C}$ fiel $\mu$ jedoch wieder ab. Dies stimmt mit dem früher untersuchten und durch neue Messungen bestätigten Verhalten von Rubidium überein.
\end{abstract}

Die Isotopenüberführung in flüssigen Metallen nimmt im allgemeinen mit der Temperatur zu (In, $\mathrm{Ga}, \mathrm{Sn}, \mathrm{Hg})^{1-5}$. Jedoch wurde kürzlich $^{6}$ ein Hinweis dafür erhalten, daß sie in $\mathrm{Rb}$ ein Maximum durchläuft. Zur Prüfung dieses Befundes wurden weitere Versuche an $\mathrm{K}$ und $\mathrm{Rb}$ vorgenommen, die in der Tat Temperaturen maximaler Isotopenüberführung ergaben, und zwar für $\mathrm{K}$ bei ca. $270{ }^{\circ} \mathrm{C}$ und für Rb bei ca. $180{ }^{\circ} \mathrm{C}$.

\section{Experimentelles}

Das Metall (Reinheit: >99,9\%) wurde in Kapillarapparaturen Gleichstrom ausgesetzt. Die Versuchsanordnung war der früher ${ }^{6}$ für $\mathrm{Rb}$ angewandten ähnlich. Bei einem Versuch mit K und einem Versuch mit $\mathrm{Rb}$ hatte jedoch die Apparatur auch einen

1 E. HaffFNer, Nature, Lond. 172, 775 [1953].

2 A. Lodding, Z. Naturforschg. 12 a, 569 [1957].

3 A. Klemm, Proc. Isotope Sep., Amsterdam 1957.

4 A. Klemm, Z. Naturforschg. 9 a, 1031 [1954].

5 A. Lodding, A. LundÉn u. H.v. Ubisch, Z. Naturforschg. 11 a, 139 [1956]. - M. Goldman, G. Nief u. E. Roth, C. R. Acad.
Anodenschenkel. Der letztere Versuch wurde nach 2 Tagen durch einen Abbruch in der Metallsäule bei der Anode beendet. Weitere 2 „Zweischenkel-Versuche“ mit Rb und 5 „Zweischenkel-Versuche“ (über $150{ }^{\circ} \mathrm{C}$ ) mit $\mathrm{K}$ mißlangen nach noch kürzerer Zeit durch Bruch an der Anode. Dies kann vielleicht der Elektroosmose (siehe Anm. ${ }^{\boldsymbol{\tau}}$ ) zugeschrieben werden. Möglicherweise spielt auch die mit einem BeckmanFlammenphotometer festgestellte Tatsache eine Rolle, $\mathrm{da} ß$ das beigemengte Natrium zur Anode überführt wird. Es könnte dort das Glas mehr als Rb oder $\mathrm{K}$ angreifen.

Bei Temperaturen oberhalb ca. $180{ }^{\circ} \mathrm{C}$ bei $\mathrm{Rb}$ und ca. $220^{\circ} \mathrm{C}$ bei $\mathrm{K}$ wurden die Kapillarwände sichtbar angegriffen. Bei dem Herauslösen des Metalls aus den Rohrstümpfen nach den Versuchen folgte ein in $\mathrm{HNO}_{3}$ unlöslicher Schlamm mit.

Sci., Paris 243, 1414 [1956]. - I. V. Bogojavlenskij, V. N. Grigorjev, N. S. Rudenko u. D. G. Dolgopolov, Sov. Phys. 6, 450 [1958] und J. Exp. Theor. Phys., USSR 33, 581 [1957].

6 A. Lodding, Z. Naturforschg. 14 a, 7 [1959].

7 A. KLeMm, Z. Naturforschg. 13 a, 1039 [1958]. 